\title{
Limited immune tolerance induced by transient mixed chimerism
}

\author{
Ji Hyun $\mathrm{Yu}^{1}$, Byung Ha Chung ${ }^{1}$, Eun Ji Oh³ ${ }^{3}$ Ji-Il Kim, Hee-Je Kim², In Sung Moon ${ }^{4}$, and Chul Woo Yang ${ }^{1}$
}

${ }^{1}$ Division of Nephrology, Department of Internal Medicine, ${ }^{2}$ Department of Hematology, Catholic Blood and Marrow Transplantation Center, Departments of ${ }^{3}$ Laboratory Medicine and ${ }^{4}$ Surgery, College of Medicine, Seoul St. Mary's Hospital, The Catholic University of Korea, Seoul, Korea

Received: October 25, 2014 Revised : November 17, 2014 Accepted: November 27, 2014

\section{Correspondence to}

Chul Woo Yang, M.D.

Department of Internal

Medicine, College of Medicine, Seoul St. Mary's Hospital, The

Catholic University of Korea, 222 Banpo-daero, Seocho-gu, Seoul 06591, Korea

Tel: +82-2-2258-6851

Fax: +82-2-2258-6917

E-mail:yangch@catholic.ac.kr
To the Editor,

The short-term clinical outcomes of kidney transplantation (KT) have been improved significantly with advances in drugs for the induction and maintenance of immunosuppression and the development of immune-monitoring methods for sensitization and rejection [1]. However, chronic use of immunosuppressive agents can result in infection, malignancy, and metabolic derangement associated with cardiovascular complications [2]. Moreover, currently used immune suppressants do not prevent chronic rejection, which is the most important cause of late allograft loss [3].

Over the last several years, immune tolerance induction to overcome such complications or limitations of immune suppressants has been attempted in solid organ transplantation. Indeed, successful cases of immune tolerance induction through mixed chimerism after combined hematopoietic stem cell transplantation (HSCT) and KT have been reported in a few centers $[4,5]$. However, there are still many obstacles and limitations to the combined HSCT and KT protocols in current use, and long-term immune tolerance was found only in a minor proportion of the patients in those trials.

Here, we introduce our experiences with a method to achieve immune tolerance through the formation of mixed chimerism after combined HSCT and KT from a human leukocyte antigen (HLA)-haploidentical sibling donor. This trial was approved by the Institutional Review Board of Seoul St. Mary's Hospital (KC12EISEo635).

A 40-year-old woman with end-stage renal disease caused by focal segmental glomerulosclerosis was admitted for KT. Her potential donor was an HLA-haploidentical brother. Panel reactive antibody was $\% \%$ for both class I and II, and crossmatch tests were negative for $\mathrm{T}$ and $\mathrm{B}$ cells.

Our center's protocol for immune tolerance induction is shown in Fig. 1. Briefly, beginning 18 days before transplantation, the donor received granulocyte-colony stimulating factor at $10 \mu \mathrm{g} / \mathrm{kg}$ subcutaneously for 5 days. $\mathrm{CD}_{34}{ }^{+}$-enriched peripheral blood stem cells were harvested, and peripheral $\mathrm{T}$ cells were depleted using a Clini-MACS bead kit (Miltenyi Biotec, Bergisch Gladbach, Germany) and cryopreserved until they were infused. The recipient was admitted 7 days before transplantation, and fludarabine was started at a dose of $30 \mathrm{mg} / \mathrm{m}^{2}$ for 4 days (days -6 to -3 ). Antithymocyte globulin was infused at $2.5 \mathrm{mg} / \mathrm{kg}$ for 2 days (days -4 to -3 ), and total nodal irradiation (750 cGy) was performed on day -2. For infection prophylaxis, fluconazole, sulfamethoxazole/trimethoprim, and acyclovir were used from day -5 to 6 months posttransplantation. 


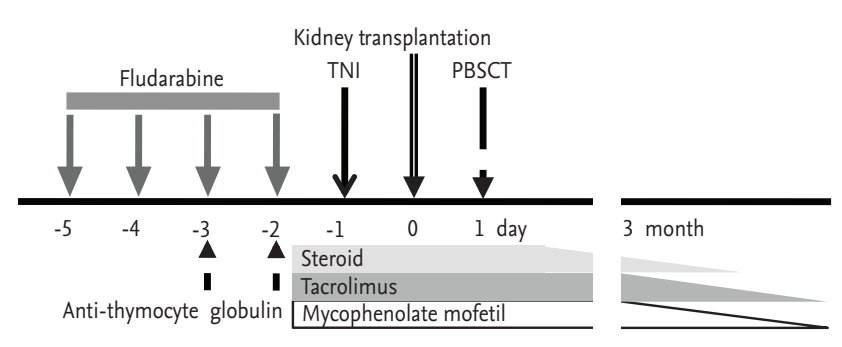

Figure 1. Immune tolerance induction protocol. The conditioning regimen consisted of fludarabine $(30 \mathrm{mg} / \mathrm{m} 2)$ administered intravenously for 4 days (from day -5 to -2 ), antithymocyte globulin $(2.5 \mathrm{mg} / \mathrm{kg})$ administered intravenously for 2 days (on day -3 and -2 ), and total nodal irradiation (TNI, 750 cGy). Tacrolimus (target trough level of 8 to $12 \mathrm{ng} / \mathrm{mL}$ ), steroid (methylprednisolone $125 \mathrm{mg}$ once a day on day $-2,125$ $\mathrm{mg}$ bid on day $-1,125 \mathrm{mg}$ four times a day on day o, then tapered thereafter) and mycophenolate mofetil (1.5 g/day) were started from day -2 . On day o, the kidney was transplanted, and on day 1 , cryopreserved peripheral blood stem cells $(4.42$ $\times 10^{6} \mathrm{cells} / \mathrm{kg}$ ) were infused intravenously. PBSCT, peripheral blood stem cell transplantation.

Immunosuppressants, namely tacrolimus (target trough blood level of 8 to $12 \mathrm{ng} / \mathrm{mL}$ ), mycophenolate mofetil (1.5 $\mathrm{g} /$ day), and steroids were started 2 days before KT (as in conventional KT). The collected donor peripheral blood stem cells were infused the following day.

Our immune monitoring protocol was as follows. First, chimerism was measured every week for 1 month and every 3 months thereafter by DNA genotyping of simple sequence-length polymorphic markers that encoded short tandem repeats. Second, the mixed lymphocyte reaction (MLR), an ImmuKnow study, and immune cell monitoring were performed before transplantation, every week for 1 month posttransplantation, and every 3 months thereafter. Third, protocol kidney biopsies were scheduled 3 and 6 months after KT and just before cessation of the immunosuppressive agent. We planned to taper the immunosuppressant dose beginning 3 months after KT and to discontinue the immunosuppressants within 1 year after KT.

After transplantation, the renal allograft and bone marrow function recovered well (Fig. 2). Additionally, multilineage mixed chimerism developed successfully and was maintained until 8 weeks after KT (Fig. 3A). Even after the disappearance of chimerism, a one-way MLR still showed a low response to the donor, implying sustained suppression of donor-specific allo-immune responses (Fig. 3B). During the follow-up period, the pa-

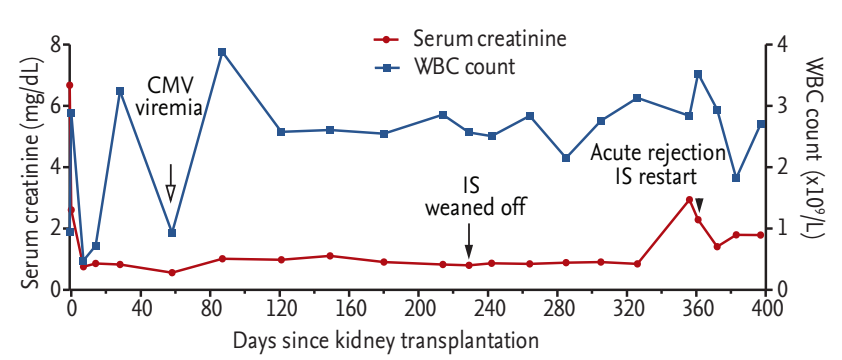

Figure 2. Clinical course of the patient. The serum creatinine level (red) decreased immediately after kidney transplantation (KT) and was maintained below $1 \mathrm{mg} / \mathrm{dL}$ until 127 days after discontinuation of the immunosuppressants (ISs) (arrow, discontinuation of immunosuppression; arrowhead, immunosuppression restart). The white blood cell (WBC) count (blue) recovered for 1 month after KT, and except for a period of cytomegalovirus (CMV) viremia (hollow arrow), was maintained above $2 \times 10^{9}$ cells/L.

tient did not experience graft-versus-host disease or any serious infectious complication, except for an episode of cytomegalovirus viremia accompanied by transient pancytopenia 2 months after transplantation.

Beginning 3 months after KT, we gradually reduced the dose of the immunosuppressant and discontinued it at 229 days after noticing a low donor-specific response in the MLR. We did not perform protocol biopsies because of thrombocytopenia. However, a kidney biopsy performed 44 days after discontinuation of immunosuppression (i.e., 273 days after KT) showed no evidence of acute rejection. At that time, the MLR still showed a low response to the donor. At 127 days after discontinuation of immunosuppressant (356 days after $\mathrm{KT}$ ), the serum creatinine level increased abruptly from 0.85 to $2.94 \mathrm{mg} / \mathrm{dL}$, and a kidney biopsy showed acute $\mathrm{T}$ cell-mediated rejection, type IIA. Donor-specific anti-HLA antibody was not detected. We began steroid pulse therapy followed by antithymocyte globulin administration and initiated maintenance immunosuppression again 366 days after KT (137 days after discontinuation of immunosuppression). After the antirejection treatment, the serum creatinine level decreased to $1.41 \mathrm{mg} / \mathrm{dL}$ and was maintained stably until 407 days after KT.

In this case, mixed chimerism was achieved successfully and lasted for 8 weeks after combined KT and HSCT. The MLR showed a sustained low response to 

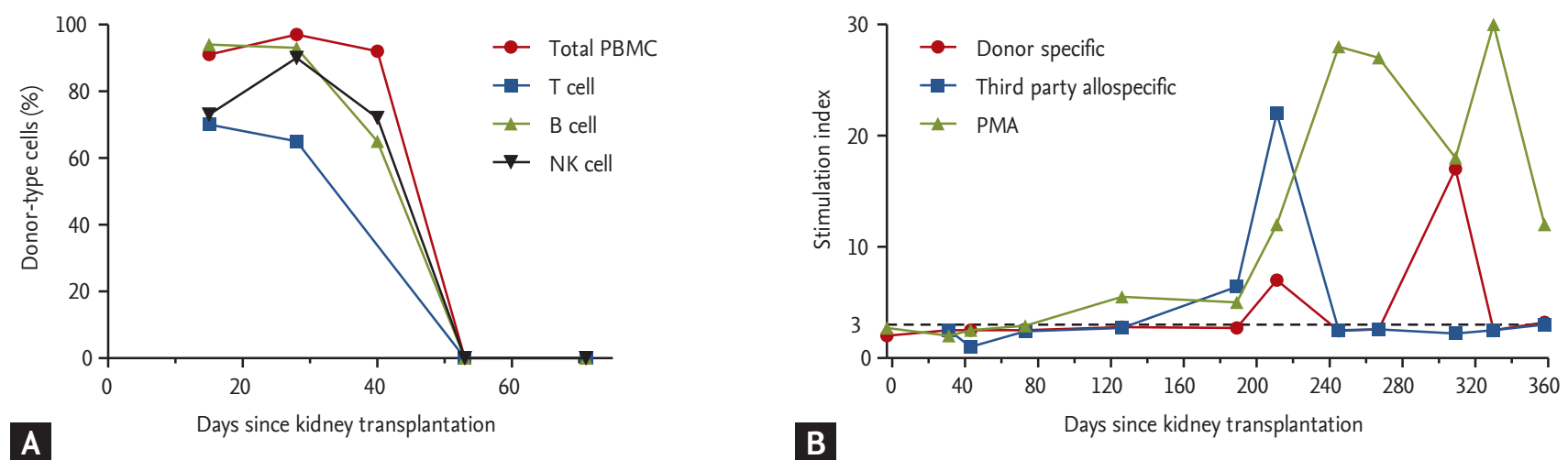

Figure 3. Results of immune monitoring. (A) The percentage of donor chimerism in the total peripheral blood mononuclear cells (PBMCs, red), T cells (blue), B cells (green), and natural killer (NK) cells (black) indicates that successful mixed chimerism was achieved, but it disappeared 53 days after kidney transplantation. Mixed lymphocyte reaction showed a sustained low response to the donor (red) after the disappearance of mixed chimerism (8 weeks after KT). (B) There was a transient increase in the donor-specific $\mathrm{T}$ cell response around 320 days after KT, but it was transient and decreased again. A stimulation index of $<3$ means no response. PMA, phorbol myristate acetate.

the donor even after mixed chimerism disappeared, lasting for 12 months. Additionally, an allograft biopsy performed 44 days after discontinuation of the immunosuppressant showed no evidence of rejection, and the allograft function was stable until 4 months after immunosuppression was stopped. During the follow-up period, the patient had no donor-specific anti-HLA antibodies. Taken together, all of these findings suggest that immune tolerance was successfully induced temporarily. However, this state was not permanent, and the patient required maintenance immunosuppression again 4 months after the discontinuation of immunosuppressants.

The failure to maintain long-term immune tolerance in this case requires consideration of two issues. The first is the role of mixed chimerism in the maintenance of immune tolerance. It is evident that development of mixed chimerism is essential for the induction of early-stage immune tolerance in solid organ transplantation. However, whether durable mixed chimerism is necessary for permanent immune tolerance is still debated. In a previous report from Massachusetts General Hospital [5], an immune-tolerant state was maintained despite transient mixed chimerism. The proposed mechanism for that was the upregulation of a regulatory $\mathrm{T}$ cell population with suppressive capacity and peripheral deletion of donor-specific T cells by the transplanted renal allograft. In contrast, another group proposed that durable mixed chimerism was essential for the long-term maintenance of immune tolerance [4]. Thus, in this case, whether the disappearance of mixed chimerism was significantly associated with the loss of immune tolerance or other unknown mechanisms are involved remains unclear. Indeed, the underlying mechanism for the maintenance of immune tolerance remains to be fully determined.

The second issue that needs to be considered is whether a monitoring tool to measure immune tolerance is needed. As described above, detection of mixed chimerism does not always coincide with an immune-tolerant state because immune tolerance may persist after the disappearance of macrochimerism [5]. However, there is no available method to confirm the state of immune tolerance after the disappearance of mixed chimerism. In this case, we suspected that immune tolerance was maintained for 9 months, even after the disappearance of mixed chimerism. However, the patient required maintenance immunosuppression again due to allograft rejection, despite the fact that the MLR still showed a low donor-specific allo-immune response. If we possessed a tool to accurately measure immune tolerance, we could have determined the time point at which the tolerance state was broken and thus could have started immunosuppressive therapy before the development of rejection.

In summary, we have reported a case in which im- 
mune tolerance was successfully induced by the development of mixed chimerism using combined HSCT and KT. However, the mixed chimerism and immune tolerance were not durable, and the patient returned to maintenance immunosuppression 137 days after discontinuation of the initial immunosuppression therapy. This case suggests that future studies should focus on the development of an effective induction protocol for more stable immune tolerance and monitoring methods to accurately measure immune tolerance.

Keywords: Immune tolerance; Kidney transplantation; Chimerism

\section{Conflict of interest}

No potential conflict of interest relevant to this article was reported.

\section{Acknowledgments}

This study was supported by the Research Fund of Seoul St. Mary's Hospital, College of Medicine, The Catholic University of Korea and by a grant from the
Korean Health Technology R\&D Project, Ministry for Health and Welfare, Republic of Korea (HIo9C1555).

\section{REFERENCES}

1. Durrbach A, Francois H, Beaudreuil S, Jacquet A, Charpentier B. Advances in immunosuppression for renal transplantation. Nat Rev Nephrol 2010;6:160-167.

2. Pascual M, Theruvath T, Kawai T, Tolkoff-Rubin N, Cosimi AB. Strategies to improve long-term outcomes after renal transplantation. N Engl J Med 2002;346:580-590.

3. Sellares J, de Freitas DG, Mengel M, et al. Understanding the causes of kidney transplant failure: the dominant role of antibody-mediated rejection and nonadherence. Am J Transplant 2012;12:388-399.

4. Leventhal J, Abecassis M, Miller J, et al. Tolerance induction in HLA disparate living donor kidney transplantation by donor stem cell infusion: durable chimerism predicts outcome. Transplantation 2013;95:169-176.

5. Kawai T, Sachs DH, Sprangers B, et al. Long-term results in recipients of combined HLA-mismatched kidney and bone marrow transplantation without maintenance immunosuppression. Am J Transplant 2014;14:1599-1611. 